\title{
High Order Sliding Mode Observer-Based Output Feedback Controller Design For Electro-Hydraulic System
}

\author{
Shibly Ahmed AL-Samarraie* Mustafa H. Mishary** \\ *,** Department of Control and Systems/University of Technology \\ *Email: 60132@uotechnology.edu.iq \\ **Email: albmustafa@yahoo.com
}

(Received 7 March 2016; accepted 15 June 2016)

http://dx.doi.org/10.22153/kej.2016.06.002

\begin{abstract}
A perturbed linear system with property of strong observability ensures that there is a sliding mode observer to estimate the unknown form inputs together with states estimation. In the case of the electro-hydraulic system with piston position measured output, the above property is not met. In this paper, the output and its derivatives estimation were used to build a dynamic structure that satisfy the condition of strongly observable. A high order sliding mode observer (HOSMO) was used to estimate both the resulting unknown perturbation term and the output derivatives. Thereafter with one signal from the whole system (piton position), the piston position make tracking to desire one with a simple linear output feedback controller after canceling the perturbation term.

The numerical simulation results showed excellent performance of the proposed output feedback controller in forcing the piston position to follow the desired reference position. Moreover, the control effort spent was minimal.
\end{abstract}

Keywords: Electro-Hydraulic system, Strong observability, Unmatched disturbance, High order sliding mode observer.

\section{Introduction}

In many tracking or regulating problem design, it is impractical to assume that all the system phase variables are measured [1, Chapter 7]. The problem of states reconstruction together with perturbation (unknown inputs and parametric uncertainties) estimation is one of the most important studies in modern control theory [2].

The computation of system states and perturbation can be done by a dynamical system called robust observer [1, Chapter 7].

For perturbed linear system the traditional Luenberger observer derived the state estimation error to bounded region around the origin [3, Chapter 7]. In addition to that the estimation error with sliding mode observer based on first order sliding mode converge asymptotically to the origin if the measured outputs have relative degree one with respect to the perturbation term [3, Chapter 3]. But sliding mode observer based on high order sliding mode skip the need to the relative degree one, because this observer include a robust (exact with measurement noise free) differentiator [3, Chapter 7]. The relative degree one with respect to the perturbation is a restriction to design an observer [4].

As a literature review, the performance improvement of the sliding mode control for the electro hydraulic system (EHS) position tracking was studied in [5] such that the dynamic friction model of the EHS was inserted in the simulation model instead of the one that to be controlled. An approximate ideal sliding mode control with varying boundary layers for EHS was proposed by [6] to improve the position tracking performance such that the experimental results have shown robustness against the various set points. By combining the sliding mode control and a simple robust method, the adaptive sliding mode control for the EHS was presented by [7] such that the results have shown a good performance of the position tracking. 
Observer based back stepping control design for the EHS was studied in $[8,9]$ such that the proposed controller was designed in the presence of both friction and load force where the ultimate boundedness of the position tracking error was guaranteed.

In [10] a nonlinear model of the EHS dynamical model have been developed such that a robust $H_{\infty}$ position control extended with integral action was proposed, where the experimental results have shown the robustness of the closed loop system against the parametric perturbation and the load force.

HOSMO with exact states and unknown bounded inputs observation for strongly observable system was proposed by [2]. In [11] the states and the unknown unbounded (but only requires boundedness of at least one of their high order derivatives) inputs were estimated. A global finite time exact observation of the state vector for general (the measured outputs are perturbed by the same unknown form of the system dynamics with dimension of the unknown input vector less than the number of the measured outputs) strongly observable linear time invariant systems with bounded unknown Lebesgue measurable inputs was proposed by [12].

The organization of this paper is as follows; the formulation of the problem statement is presented in section 2, while the HOSMO based controller deign is introduced in section 3. In section 4 the Electro-hydraulic system takes as a case study, while the simulation results and discussions are developed in section 5 .

\section{Problem Statement}

Consider a dynamical system described by

$$
\left.\begin{array}{c}
\dot{x}_{1}=x_{2} \\
\dot{x}_{2}=f_{1}\left(x_{1}, x_{2}\right)+g_{1}\left(x_{1}, x_{2}\right) x_{3} \\
+d_{1}\left(x_{1}, x_{2}, t\right) \\
\dot{x}_{3}=f_{2}\left(x_{1}, x_{2}, x_{3}\right)+g_{2}\left(x_{1}, x_{2}, x_{3}\right) u \\
\begin{array}{c}
y=d_{2}\left(x_{1}, x_{2}, x_{3}, t\right) \\
y=
\end{array}
\end{array}\right\}
$$

where, $d_{1}\left(x_{1}, x_{2}, t\right)$ and $d_{2}\left(x_{1}, x_{2}, x_{3}, t\right)$ are the mismatched and matched unknown perturbation forms. The perturbation includes parametric uncertainties and the unknown bounded external inputs with knowing that that both $f_{1}$ and $d_{1}\left(x_{1}, x_{2}, t\right)$ are assumed to be locally Lipschitz. In addition $y$ is assumed here to be the only measured output.

The observability problem can be regarded as follows; the possibility to build a new dynamic system called robust observer depends only on given information called measured outputs used to reconstruct both of system states and unknown form [13, Chapter 1]. The strong observability, that is required observability condition, confirms the existence of transformation matrix that transformed the original form dynamic to a phase variable canonical form such that there is a robust observer which is exact with measurement noise free to reconstruct both of states and the unknown form, such observer is a HOSMO [3, Chapter 7]. From an observer design point of view (Eq. (1)) one cannot design an observer which used to reconstruct the unknown form along with states estimation due to the relative degree condition which is not satisfied here. The electro-hydraulic system is an example described mathematically by Eq. (1).

In modern control system the reduction of sensors devices produce less measurement noise, such that the proposed controller become more accurate in addition to cost reduction[2].

Now consider the following assumption; Assumption (1): the right hand side of $\dot{x}_{2}$ in Eq. (1) is a differentiable function, i.e., $f_{1}\left(x_{1}, x_{2}\right)$, $g_{1}\left(x_{1}, x_{2}\right) x_{3}$ and $d_{1}\left(x_{1}, x_{2}, t\right)$ are smooth functions of $\left(x_{1}, x_{2}, t\right)$.

Based on the above assumption, Eq. (1) can be transformed to a phase variable canonical form. As a result it can be obtained a strongly observable system such that the state variables vector is the output measurement and its derivatives. Thereafter HOSMO will be adapted here to reconstruct the resulting new unknown form together with new state variable vector estimation. The new unknown form means that a perturbation term resulted from differentiating $\dot{x}_{2}$. In addition to that the new unknown form of the resulting dynamic (canonical transformed form of Eq. (1)) satisfies the matching condition where after estimating this form one can cancel it and then use any successful linear controller to drive $y=x_{1}$ to track desired one.

The goal of this paper is to build a system dynamic related to the system dynamic as in Eq. (1) and transform it to a phase variable canonical form via HOSMO. Then a linear state feedback will be designed to derive the output to follow the desired reference.

\section{High Order Sliding Mode Observer Based Controller Design}

Keeping in mind the dynamic of Eq. (1) and denoting that: 
$\gamma_{1}=y=x_{1}, \quad \gamma_{2}=\dot{y}=x_{2}, \quad \gamma_{3}=\ddot{y}=\dot{x}_{2}$

Now by considering assumption (1) the model dynamic in Eq. (1) can be rewritten as follows;

$\dot{\gamma}=A \gamma+B u+D \delta\}$

$y=C \gamma$

Where:

$\gamma=\left[\begin{array}{l}\gamma_{1} \\ \gamma_{2} \\ \gamma_{3}\end{array}\right], A=\left[\begin{array}{lll}0 & 1 & 0 \\ 0 & 0 & 1 \\ 0 & 0 & 0\end{array}\right], B=\left[\begin{array}{c}0 \\ 0 \\ g_{1} g_{2}\end{array}\right]$

$D=\left[\begin{array}{l}0 \\ 0 \\ 1\end{array}\right], C=\left[\begin{array}{lll}1 & 0 & 0\end{array}\right]$

$\delta=g_{1}\left(f_{2}+d_{2}\right)+\frac{d}{d t}\left(f_{1}+d_{1}\right)$

where $\left(f_{1}+d_{1}\right)$ is assumed differentiable as mentioned above. Therefore it can be seen that the dynamic in Eq. (3) is strongly observable (see Appendix A). The next step is devoted to design a HOSMO to reconstruct the perturbation $\delta$ together with estimating the new state $\gamma$. It consists of building a standard Luenberger observer together with a high order sliding mode differentiator.

A standard Luenberger observer for the system in Eq. (3) is given by

$$
\begin{aligned}
\dot{z} & =A z+B u+L(y-C z) \\
& =A z+B u+L(C \gamma-C z)
\end{aligned}
$$

where $z \in \mathcal{R}^{3}$. Define: $e=\gamma-z$, then from Eq. (3) and Eq. (4) we obtain:

$$
\left.\begin{array}{l}
\dot{e}=(A-L C) e+D \delta \\
e_{y}=C e
\end{array}\right\}
$$

where $e_{y}=y-C z=C \gamma-C z=C e$.

The traditional Luenberger observer with $\delta=0$ ensures the asymptotic convergence to zero for the estimation error due to that $(A-L C)$ is Hurwitz [14] without the need to differentiating the output n-1 times. But with unknown bounded $\delta$ the Luenberger observer providing a bounded estimation error [3]. A high order sliding mode differentiator companied with Luenberger observer confirms a finite time convergence of the resulting estimation error to zero as follow [3]:

$\left[\begin{array}{l}e_{y} \\ \dot{e}_{y} \\ \ddot{e}_{y} \\ \ddot{e}_{y}\end{array}\right]=\left[\begin{array}{c}C \\ C(A-L C) \\ C(A-L C)^{2} \\ C(A-L C)^{3}\end{array}\right] e+\left[\begin{array}{c}0 \\ 0 \\ 0 \\ C(A-L C)^{2} D\end{array}\right] \delta$

From Eq. (6), the state $\gamma$ and the perturbation $\delta$ are estimated as follows;

$\gamma=z+\left[\begin{array}{c}C \\ C(A-L C) \\ C(A-L C)^{2}\end{array}\right]^{-1}\left[\begin{array}{c}e_{y} \\ \dot{e}_{y} \\ \ddot{e}_{y}\end{array}\right]$

$\delta=\frac{1}{C(A-L C)^{2} D}\left(\dddot{e}_{y}-C(A-L C)^{3} e\right)$

Equation (7) shows that, $(\gamma-z) \rightarrow 0$ when $e_{y} \rightarrow 0$. That means a Luenberger observer is sufficient with $\delta=0$. To this end, the derivatives of $e_{y}$ to the third order are the basic implementation requirement of the above observer.

A high order sliding mode differentiator is utilized in this work to get an exact (robust with noisy measurements) $3^{\text {th }}$ order differentiation of signal $e_{y}$ as follow; Assume that the $3^{\text {rd }}$ derivative has a known Lipschitz constant $M>$ $\left|C A^{2} D\right||\delta|_{\max }\left[3\right.$, Chapter 7]. Since $C A^{2} D=1$ (see Eq. (3)), thus $M>|\delta|_{\max }$. The HOSMO dynamics is given by;

$$
\begin{aligned}
& \dot{v}_{1}=-\lambda_{4} M^{\frac{1}{5}}\left|v_{1}-e_{y}\right|^{\frac{4}{5}} \operatorname{sgn}\left(v_{1}-e_{y}\right)+v_{2} \\
& \dot{v}_{2}=-\lambda_{3} M^{\frac{1}{4}}\left|v_{2}-\dot{v}_{1}\right|^{\frac{3}{4}} \operatorname{sgn}\left(v_{2}-\dot{v}_{1}\right)+v_{3} \\
& \dot{v}_{3}=-\lambda_{2} M^{\frac{1}{3}}\left|v_{3}-\dot{v}_{2}\right|^{\frac{2}{3}} \operatorname{sgn}\left(v_{3}-\dot{v}_{2}\right)+v_{4} \\
& \dot{v}_{4}=-\lambda_{1} M^{\frac{1}{2}}\left|v_{4}-\dot{v}_{3}\right|^{\frac{1}{2}} \operatorname{sgn}\left(v_{4}-\dot{v_{3}}\right)+v_{5} \\
& \dot{v}_{5}=-\lambda_{0} M \operatorname{sgn}\left(v_{5}-\dot{v}_{4}\right)
\end{aligned}
$$

where $\quad \lambda_{0}=1.1, \lambda_{1}=1.5, \lambda_{2}=2, \lambda_{3}=3$, $\lambda_{4}=5$ [3, Chapter 6]. From Eq. (9), Eq. (7) becomes;

$\hat{\gamma}=z+\left[\begin{array}{c}C \\ C(A-L C) \\ C(A-L C)^{2}\end{array}\right]^{-1}\left[\begin{array}{l}v_{1} \\ v_{2} \\ v_{3}\end{array}\right]$

Based on Eq. (9) and Eq. (10), Eq. (8) becomes;

$\hat{\delta}=\frac{1}{C(A-L C)^{2} D} *$

$\left\{v_{4}-C(A-L C)^{3}\left[\begin{array}{c}C \\ C(A-L C) \\ C(A-L C)^{2}\end{array}\right]^{-1}\left[\begin{array}{l}v_{1} \\ v_{2} \\ v_{3}\end{array}\right]\right\}$

By taking into consideration Eq. (10) and Eq. (11), the control law is proposed here as follows;

$u=\frac{1}{g_{1} g_{2}}\left\{u_{0}\left(\hat{\gamma}_{1}, \hat{\gamma}_{2}, \hat{\gamma}_{3}\right)-\hat{\delta}\right\}$

As a result Eq. (3) becomes

$\dot{\gamma_{1}}=\gamma_{2}$

$\dot{\gamma_{2}}=\gamma_{3}$

$\dot{\gamma_{3}}=u_{0}\left(\hat{\gamma}_{1}, \hat{\gamma}_{2}, \hat{\gamma}_{3}\right)$

where $u_{0}\left(\hat{\gamma}_{1}, \hat{\gamma}_{2}, \hat{\gamma}_{3}\right)$ can be designed as any linear states feedback controller such that the system dynamics described by (13) is globally asymptotically stable.

The control design idea presented here is depict in Fig. (1), where the piston position is the only measured output. The feedback controller was designed based on higher order sliding mode observer.

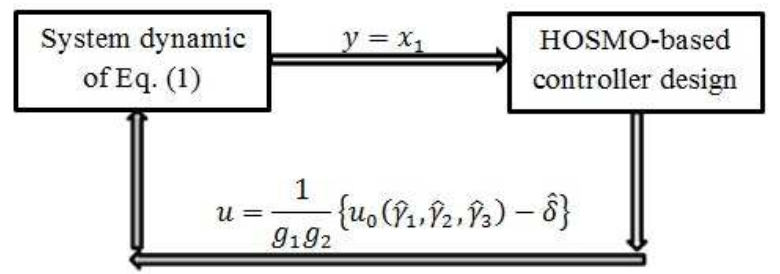

Fig. 1. Control design idea 


\section{High Order Sliding Mode Observer- Based Controller Design for Electro- Hydraulic System}

The mathematical model for electro-hydraulic system that used in this work is described by [15]; $\dot{x}_{1}=x_{2}$

$\left.\begin{array}{l}\dot{x}_{2}=f_{1}\left(x_{1}, x_{2}\right)+g_{1} x_{3}+d_{1}\left(x_{1}, x_{2}\right) \\ \dot{x}_{3}=f_{2}\left(x_{2}, x_{3}\right)+g_{2} u_{1}+d_{2}\left(u, x_{2}, x_{3}\right) \\ y=x_{1}\end{array}\right\} \ldots$

where $x_{1}$ is the piston position (meter), $x_{2}$ is the piston velocity (meter $/ \mathrm{sec}$ ), $x_{3}$ is the pressure difference $\left(N /\right.$ meter $\left.^{2}\right), u$ is the control input (the current) $(m A), f_{1}, g_{1}, f_{2}$ and $g_{2}$ are the system dynamics nominal terms (includes nominal parameters only) with $g_{1}, g_{2}>0$. In addition $d_{1}$ and $d_{2}$ are the mismatched and matched perturbations respectively. The nominal and the perturbation terms in system dynamics are given by;

$$
\left.\begin{array}{l}
f_{1}\left(x_{1}, x_{2}\right)=-\frac{1}{m}\left(k x_{1}+b x_{2}\right) \\
g_{1}=\frac{\Omega}{m} \\
f_{2}\left(x_{2}, x_{3}\right)=-\frac{4 \beta_{e} \Omega}{V_{t}} x_{2}-\frac{4 \beta_{e} c_{t m}}{V_{t}} x_{3} \\
g_{2}=\frac{4 \beta_{e} C_{d} w k_{v}}{V_{t} \sqrt{\rho}} \sqrt{P_{s}} \\
d_{1}\left(x_{1}, x_{2}\right)=\Delta f_{1}\left(x_{1}, x_{2}\right)+\Delta g_{1} x_{3} \\
d_{2}\left(x_{2}, x_{3}\right)=\Delta f_{2}\left(x_{2}, x_{3}\right)+H u \\
H=\left\{\frac{4 \beta_{e} C_{d} w k_{v}}{V_{t} \sqrt{\rho}}+\Delta\left(\frac{4 \beta_{e} C_{d} w k_{v}}{V_{t} \sqrt{\rho}}\right)\right\} * \\
\quad \sqrt{P_{s}-\operatorname{sgn}(u) x_{3}}-\frac{4 \beta_{e} C_{d} w k_{v}}{V_{t} \sqrt{\rho}} \sqrt{P_{s}}
\end{array}\right\}
$$

where: $\Delta f_{1}, \Delta g_{1}, \Delta f_{2}$, and $\Delta g_{2}$ are the uncertainty term and its assumed here that the variation in their parameters can reach $30 \%$ of their nominal values.

As in assumption (1), $f_{1}\left(x_{1}, x_{2}\right)$ and $d_{1}\left(x_{1}, x_{2}\right)$ are differentiable functions. Accordingly the electro-hydraulic system described in Eq. (14) is equivalent to canonical system form that given in Eq. (3).

Based on section 3 one can conclude the following;

i. Since the pair $(C, A)$ in Eq. (3) is observable, therefore, there is a gain matrix $L$ used to place the eigenvalues of matrix $A-L C$ to stable one [14]. Let the desired stable eigenvalues are eig $(A-L C)=(-1,-2,-3)$ then the gain matrix $L=\left[\begin{array}{lll}6 & 11 & 6\end{array}\right]^{T}$.

ii. With the aid of Eq. (4) to Eq. (11), and $M=10^{11}$, the reconstruction of the unknown form $\delta$ along with the estimation of $\gamma$ are as follow; $\hat{\gamma}=z+\left[\begin{array}{c}v_{1} \\ 6 v_{1}+v_{2} \\ 11 v_{1}+6 v_{2}+v_{3}\end{array}\right]$

$\hat{\delta}=v_{4}+6 v_{3}+11 v_{2}+6 v_{1}$

iii. The actual current control input is calculated by

$$
u=\frac{1}{g_{1} g_{2}}\left\{u_{0}\left(\hat{\gamma}_{1}, \hat{\gamma}_{2}, \hat{\gamma}_{3}\right)-v_{4}-6 v_{3}-11 v_{2}-\right.
$$$$
\left.6 v_{1}\right\} \quad \ldots(18)
$$

For Eq. (14), in spite of the complete system parametric uncertainties, the matching condition of $d_{1}\left(x_{1}, x_{2}, t\right)$ with the actual control input are not met and strong observability does not satisfied. However, with assumption (1), and the estimated states and perturbation via HOSMO, the current control action as in Eq. (18) solves all of the above challenges. Moreover one can use any state feedback controller $u_{0}\left(\hat{\gamma}_{1}, \hat{\gamma}_{2}, \hat{\gamma}_{3}\right)$ techniques to drives $\gamma_{1}=x_{1}$ to the desired position like LQR and the pole placement.

In the present work a linear state feedback controller, uses $\hat{\gamma}_{1}, \hat{\gamma}_{2}$ and $\hat{\gamma}_{3}$ and with desired negative poles values is given in Eqs. (21) to (23) in the following section.

\section{Simulation Results and Discussions}

The obtained simulation results in this work are performed using the actual dynamics system as described by Eq. (14) with the control law and the observer dynamics as given by Eq. (18) and Eq. (9) respectively. In addition the observer parameters are presented in section 4 . The initial system states and initial observer states have been taken equal to $(0.0001,0,0),(0.0001,0,0)$ respectively.

Nominal electro-hydraulic system parameters are given in the following table.

Table 1,

The system parameters [8].

\begin{tabular}{lll}
$\begin{array}{l}\text { The } \\
\text { parameter }\end{array}$ & Description & $\begin{array}{l}\text { The nominal } \\
\text { value (SI } \\
\text { units) }\end{array}$ \\
\hline$b$ & $\begin{array}{l}\text { Viscous damping } \\
\text { coefficient. }\end{array}$ & $\begin{array}{l}19.84 * 10^{3} \\
\mathrm{Ns} / \mathrm{m}\end{array}$ \\
$\Omega$ & $\begin{array}{l}\text { Ram area of the } \\
\text { cylinder. }\end{array}$ & $\begin{array}{l}5.55 * 10^{-3} \\
\mathrm{~m}^{2}\end{array}$ \\
$\mathrm{~V}_{\mathrm{t}}$ & $\begin{array}{l}\text { Total volume of the } \\
\text { cylinder and the hoses } \\
\text { between the cylinder }\end{array}$ \\
& $\begin{array}{l}\mathrm{m}^{3} \\
\text { and the servo valve. } \\
\text { Coefficient of the total } \\
\text { internal leakage of the } \\
\text { cylinder due to } \mathrm{m}^{5} / \mathrm{Ns} \\
\text { pressure. }\end{array}$ \\
\hline
\end{tabular}




\begin{tabular}{|c|c|c|}
\hline$k$ & $\begin{array}{l}\text { Effective bulk modulus } \\
\text { of spring. }\end{array}$ & $70 * 10^{3} \mathrm{~N} / \mathrm{m}$ \\
\hline$\beta_{e}$ & $\begin{array}{l}\text { Effective } \quad \text { bulk } \\
\text { modulus. }\end{array}$ & $\begin{array}{l}700 * 10^{6} \\
\mathrm{~N} / \mathrm{m}^{2}\end{array}$ \\
\hline$C d w / \sqrt{\rho}$ & $\begin{array}{l}C_{d} \text { is the discharge } \\
\text { coefficient; } \mathrm{w} \text { is the } \\
\text { spool valve area } \\
\text { gradient and } \rho \text { is the } \\
\text { fluid density. }\end{array}$ & $\begin{array}{l}3.42 * 10^{-5} \\
\mathrm{~m}^{3} \sqrt{N s}\end{array}$ \\
\hline$m$ & Mass of the load. & $20 \sim 250 \mathrm{Kg}$ \\
\hline$P_{S}$ & $\begin{array}{l}\text { Supply pressure of the } \\
\text { fluid. }\end{array}$ & $\begin{array}{l}10^{7} \\
\mathrm{~N} / \text { meter }^{2}\end{array}$ \\
\hline$k_{v}$ & $\begin{array}{l}\text { Gain of the servo- } \\
\text { valve. }\end{array}$ & 0.03 \\
\hline
\end{tabular}

From which the parameters of Eq. (15) have been obtained;

$f_{1}=-518.52 x_{1}-147 x_{2}, \quad g_{1}=4.11 * 10^{-5}$

$f_{2}=-8.88 * 10^{9} x_{2}-0.024 x_{3}, g_{2}=5.19 *$ $10^{9}$

$d_{1}=-\Delta(518.52) x_{1}-\Delta(147) x_{2}+\Delta(4.11) *$ $10^{-5} x_{3}$

$d_{2}=-\Delta(8.88) * 10^{9} x_{2}-\Delta(0.02) 4 x_{3}+H u$ $H=$

$$
\begin{array}{r}
\{1641600+\Delta(1641600)\} \sqrt{10^{7}-\operatorname{sgn}(u) x_{3}}- \\
5.19 * 10^{9}
\end{array}
$$

Where $|\Delta()| \leq \alpha\left|()_{n}\right|$, is the parameter uncertainty, ()$_{n}$ is the nominal parameter value and $0<\alpha<1$ is the uncertainty percent.

Keeping in mind that the unknown form (perturbation term) which resulted from the electro-hydraulic system based on Eq. (3) is as follow:

$$
\delta=g_{1}\left(f_{2}+d_{2}\right)+\frac{d}{d t}\left(f_{1}+d_{1}\right)
$$

In the following the simulations are performed first for the open loop in order to examine the ability of the HOSMO in estimating the first and second derivative of $\gamma_{1}=y=x_{1}$, and also estimating the perturbation term $\delta$. Second the results of the numerical simulations for the control system are presented where the control law uses the reconstruction transformed states $\hat{\gamma}$ and perturbation estimations $\hat{\delta}$ as designed in section 4.

\subsection{Open Loop System Simulation Results}

In this subsection the control input is set equal zero $u=0$. For $\alpha=0.1$ the outputs of the HOSMO are plotted in figures (2), (3) and (4) for estimating piston position, velocity and acceleration respectively while perturbation estimation is plotted in figure (5). One can be sure from these figures, that the estimating quantities converges to their actual values in an interval of time not exceeds $0.002 \mathrm{sec}$. It can be noted also that the convergence time for the estimating quantities increased sequentially with the order of estimating states.

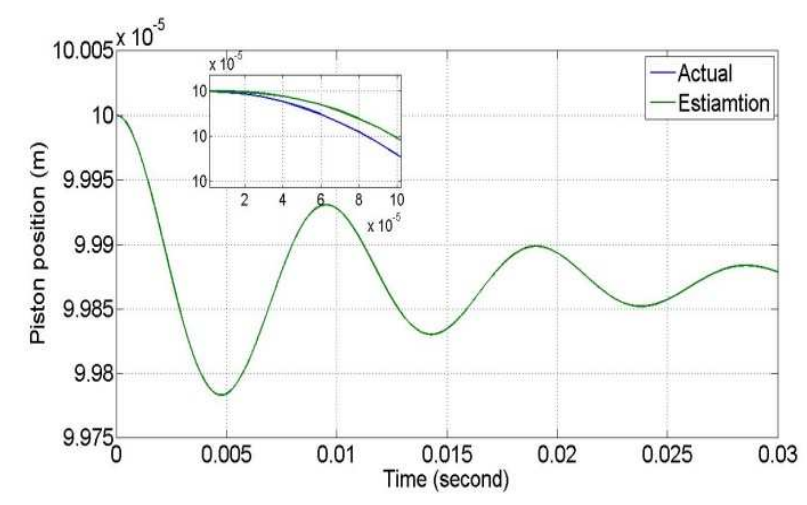

Fig. 2. Open loop piston position $x_{1}$ estimation under $\alpha=0.1$ parametric uncertainties.

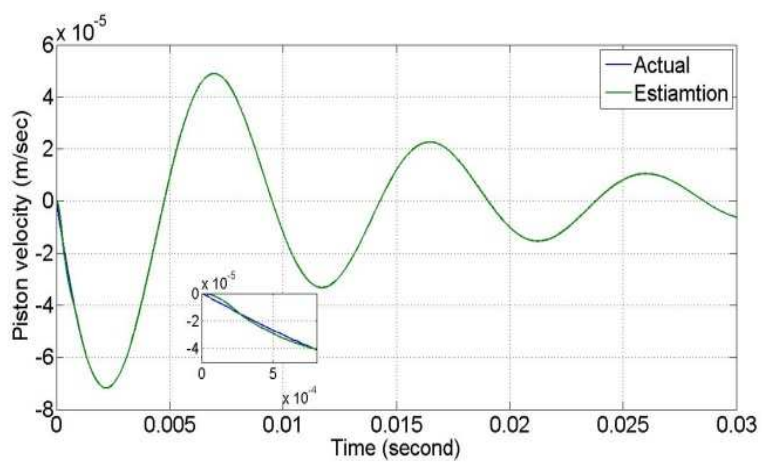

Fig. 3. Open loop piston velocity $x_{2}$ estimation under $\alpha=0.1$ parametric uncertainties.

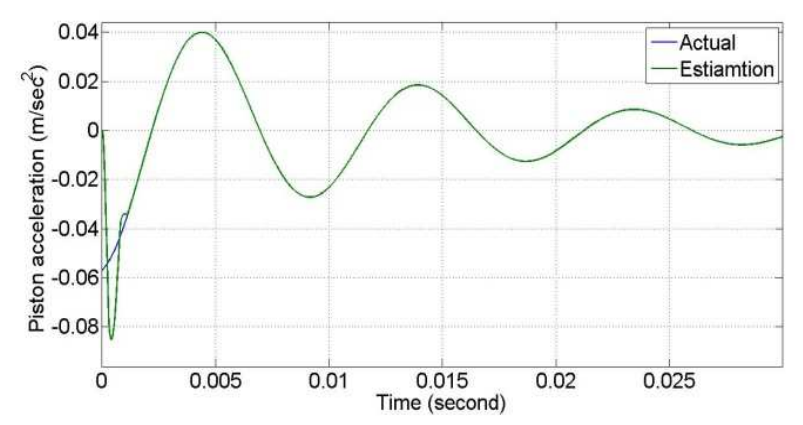

Fig. 4. Open loop piston acceleration $\dot{x}_{2}$ estimation under $\alpha=0.1$ parametric uncertainties. 


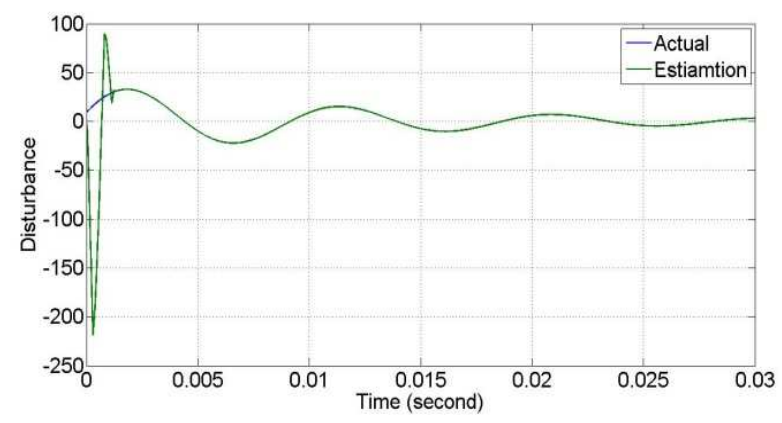

Fig. 5. Open loop $\delta$ estimation under $\alpha=0.1$ parametric uncertainties.

In order to prove the estimator robustness and the powerful of the HOSMO, the open loop simulation is repeated with $\alpha=0.3$. The estimation performance does not changed in spite of increasing the uncertainty percent $\alpha$ as can be deduced from figure (6) to figure (9).

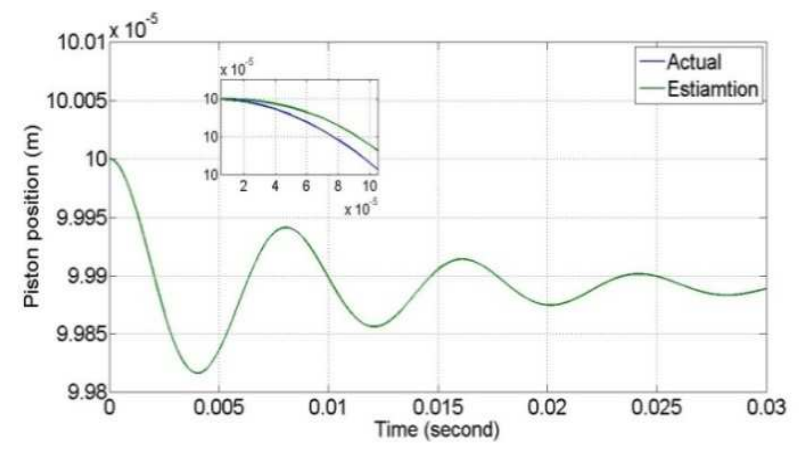

Fig. 6. Open loop piston position estimation $x_{1}$ under $\alpha=0.3$ parametric uncertainties.

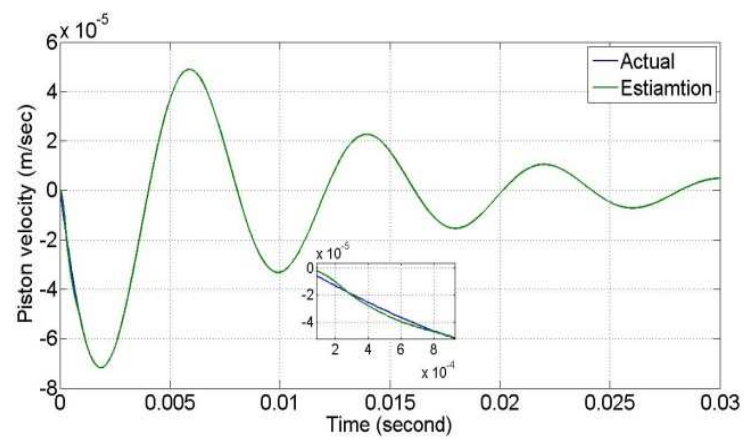

Fig. 7. Open loop piston velocity $x_{2}$ estimation under $\alpha=0.3$ parametric uncertainties.

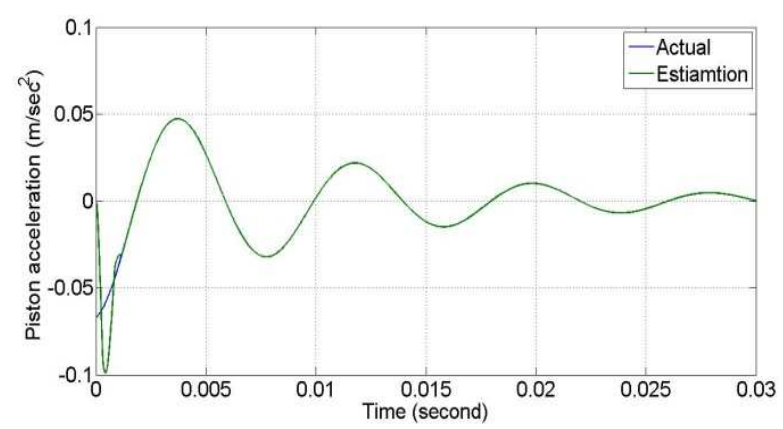

Fig. 8. Open loop piston acceleration $\dot{x}_{2}$ estimation under $\alpha=0.3$ parametric uncertainties.

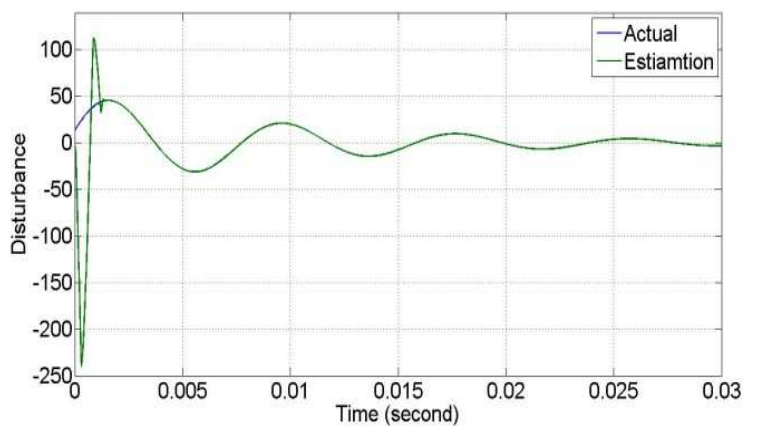

Fig. 9. Open loop $\delta$ estimation under $\alpha=0.3$ parametric uncertainties

\subsection{Closed Loop Simulation Results}

A piecewise constant and the sinusoidal are the desired piston positions which are considered in this work for the closed loop system simulation. In the following subsections the simulations are performed with $\alpha=0.3$, and the nominal controller $u_{0}\left(\hat{\gamma}_{1}, \hat{\gamma}_{2}, \hat{\gamma}_{3}\right)$ will be designed using a simple pole placement control. The proposed nominal controller that drives the piston position to continuously differentiable desired one (as in sinusoidal case) is given as follows;

$u_{0}\left(\hat{\gamma}_{1}, \hat{\gamma}_{2}, \hat{\gamma}_{3}\right)=\dddot{x}_{1 d}-K\left[\begin{array}{l}\left(\hat{\gamma}_{1}-x_{1 d}\right) \\ \left(\hat{\gamma}_{2}-\dot{x}_{1 d}\right) \\ \left(\hat{\gamma}_{3}-\ddot{x}_{1 d}\right)\end{array}\right]$

where: $x_{1 d}$ the desired piston position. While in the piecewise desired one the nominal controller is as follows;

$u_{0}\left(\hat{\gamma}_{1}, \hat{\gamma}_{2}, \hat{\gamma}_{3}\right)=-K\left[\begin{array}{l}\left(\hat{\gamma}_{1}-x_{1 d}\right) \\ \hat{\gamma}_{2} \\ \hat{\gamma}_{3}\end{array}\right]$

As mentioned earlier, one can choose feedback gains $K$ to achieve desired eigenvalues as follows; 


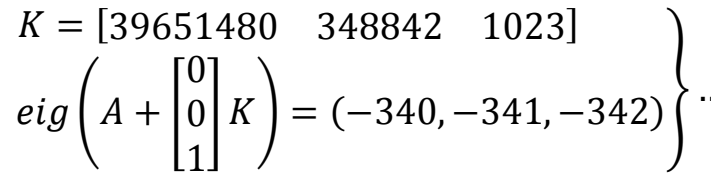

The simulation results for the closed loop control are presented in the next subsections.

\subsubsection{Piecewise Desired Piston Position Simulation Results.}

Figures (10), (11) and (12) have shown the piston position, velocity and acceleration of the electro hydraulic system respectively. It can be seen from these figures the actual piston position make tracking to desire piecewise signal with rise time approximately equal to $0.03 \mathrm{sec}$ and absolute tracking error less than $10^{-6} \mathrm{~m}$. As mentioned in the open loop results, HOSMO also have shown an accurate estimation in closed loop simulation where in these figures the blue lines represents the electro-hydraulic dynamic variables and the green ones represents the outputs of the HOSMO which were used as a states feedback in nominal controller $u_{0}\left(\hat{\gamma}_{1}, \hat{\gamma}_{2}, \hat{\gamma}_{3}\right)$.

Figure (13) refer to the pressure difference $x_{3}$ such that $x_{3} * \Omega$ represents the required hydraulic force input to drive the piston position $x_{1}$ to piecewise desired one. However, it can be seen from this figure the max value that the force input reaches approximately $\pm 28860 \mathrm{~N}$.

The estimation of the perturbation term $\hat{\delta}$ that estimated by HOSMO is clarified in figure (14) where the estimated quantity converge to actual one with time interval less than $0.01 \mathrm{sec}$. This accurate estimation was used with negative feedback signal into the actual current control input to cancel the actual effect $\delta$.

The actual current control input $u$ that used to ensure the above performance which is represented by figure (15) does not exceed $3 m A$.

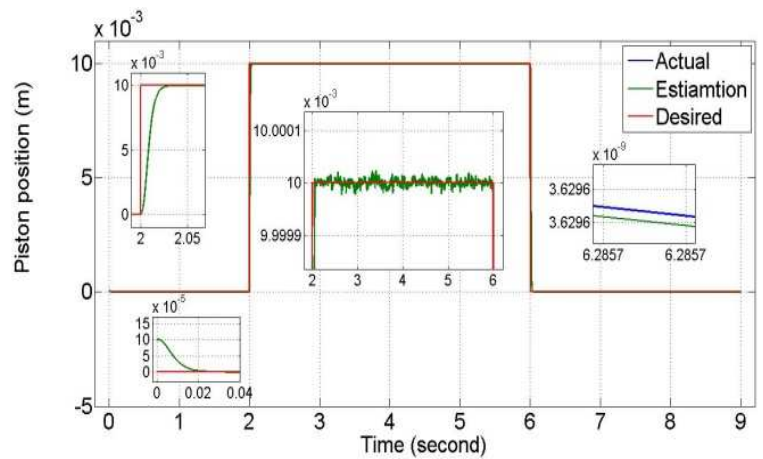

Fig. 10. Closed loop piston position $x_{1}$ under $\alpha=0.3$ parametric uncertainties tracking desired piecewise position

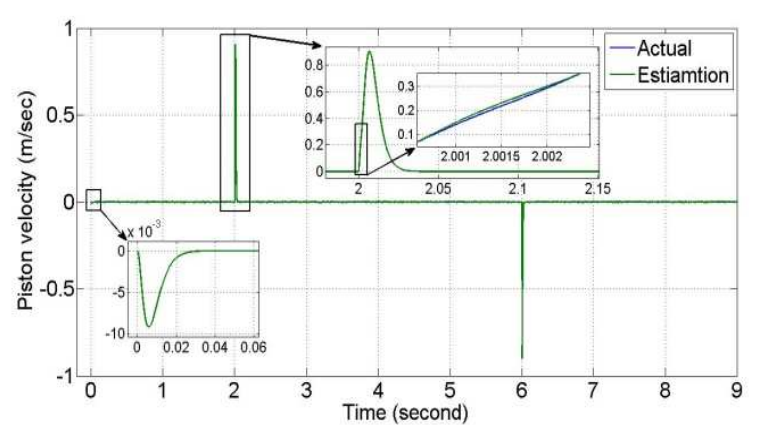

Fig. 11. Closed loop piston velocity $x_{2}$ under $\alpha=0.3$ parametric uncertainties with desired piecewise piston position.

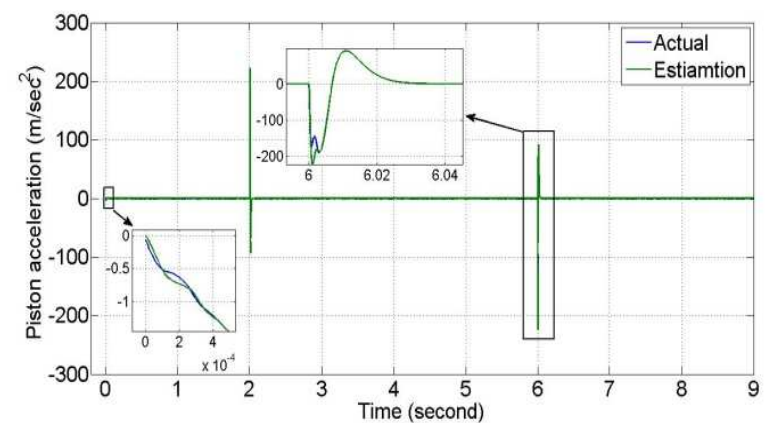

Fig. 12. Closed loop piston acceleration $\dot{x}_{2}$ under $\alpha=0.3$ parametric uncertainties with desired piecewise piston position.

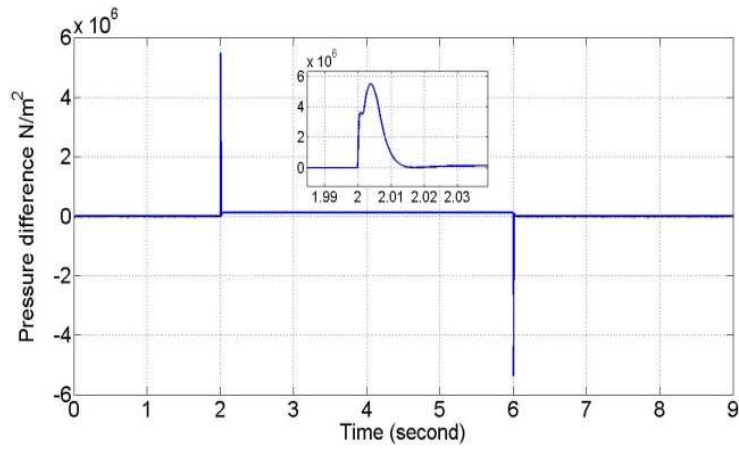

Fig. 13. Pressure difference $x_{3}$ under $\alpha=0.3$ parametric uncertainties with desired piecewise piston position.

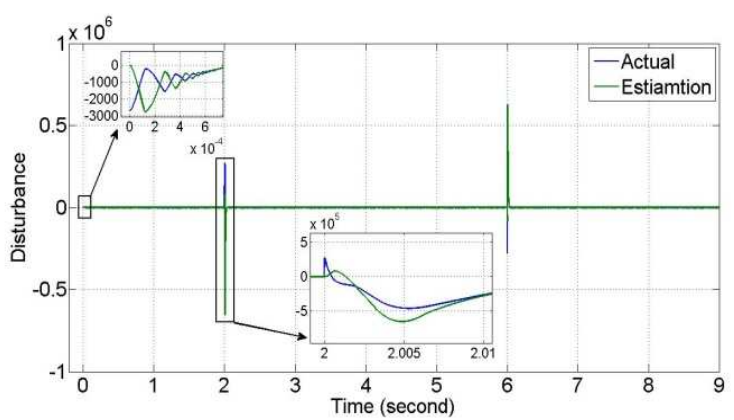

Fig. 14. Closed loop $\delta$ estimation under $\alpha=0.3$ parametric uncertainties with desired piecewise piston position. 


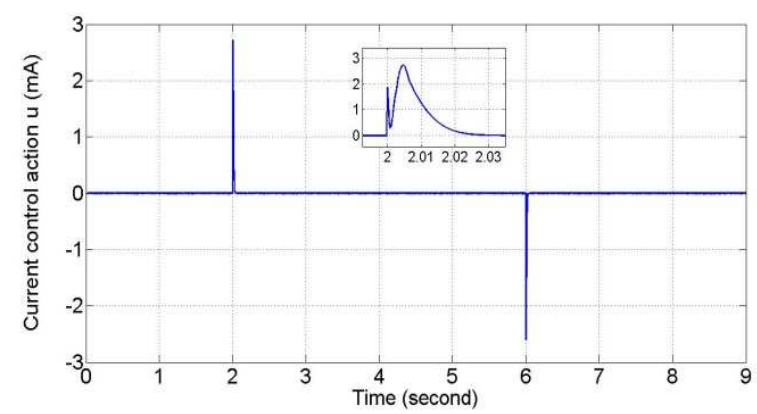

Fig. 15. Current control input under $\alpha=0.3$ parametric uncertainties with desired piecewise piston position.

\subsubsection{Sinusoidal Desired Piston Position Simulation Results.}

As in the case of piecewise desired piston position, figure (16) have shown the successful tracking of the piston position to desired one (sinusoidal) and this can be regarded as a challenge, because this tracking was constructed on the basis of knowledge of the measurement of the piston position only.

In figures (17) and (18) the actual and the estimation of the piston velocity and piston acceleration are plotted. These figures reveal the accurate estimation of these physically quantities (velocity and acceleration) via HOSMO.

The demanded hydraulic force input $x_{3} * \Omega$ in this case (sinusoidal reference) that can be noted form figure (19) oscillates between $\pm 727.05 \mathrm{~N}$.

Figures (20) and (21) show the perturbation estimation $\hat{\delta}$ and the actual current control input $u$ respectively, where the current input does not exceed $0.02 m A$.

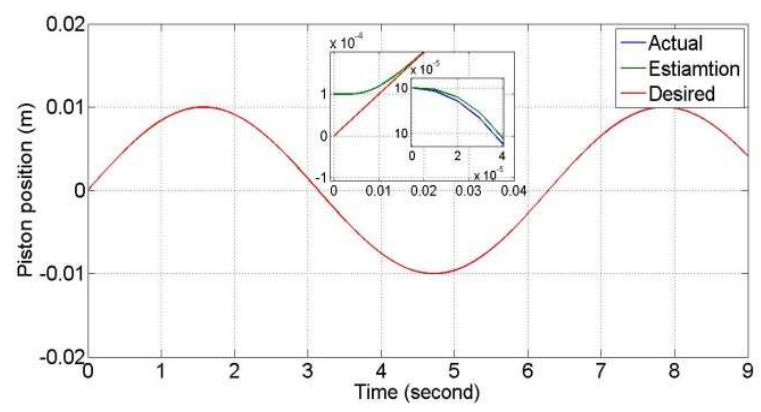

Fig. 16. Closed loop piston position $x_{1}$ under $\alpha=0.3$ parametric uncertainties tracking desired sinusoidal position.

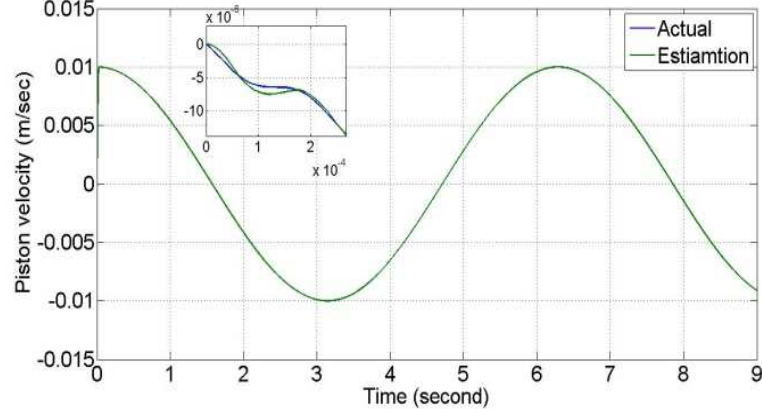

Fig. 17. Closed loop piston velocity $x_{2}$ under $\alpha=0.3$ parametric uncertainties with desired sinusoidal position.

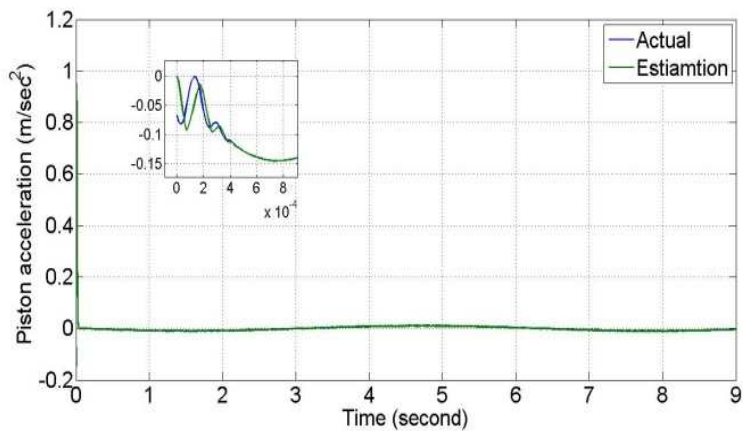

Fig. 18. Closed loop piston acceleration $\dot{x}_{2}$ under $\alpha=0.3$ parametric uncertainties with desired sinusoidal position.

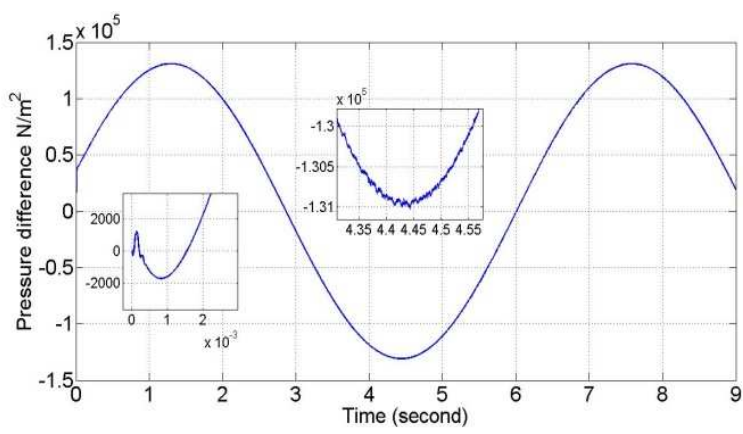

Fig. 19. Pressure difference $x_{3}$ under $\alpha=0.3$ parametric uncertainties with desired sinusoidal position.

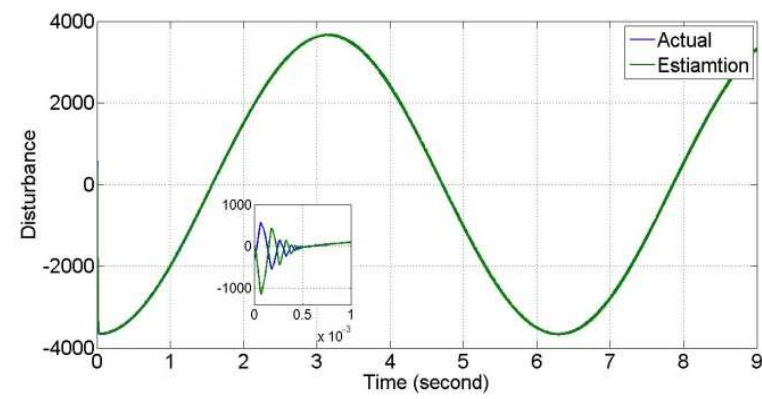

Fig. 20. Closed loop $\delta$ estimation under $\alpha=0.3$ parametric uncertainties with desired sinusoidal position. 


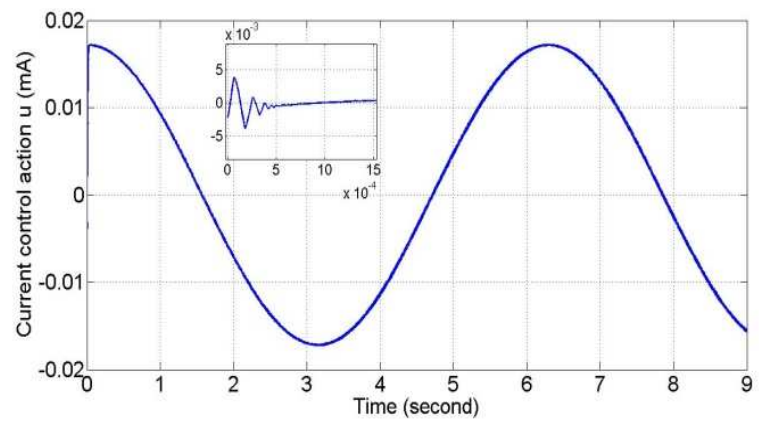

Fig. 21. Current control input under $\alpha=0.3$ parametric uncertainties with desired sinusoidal position.

From the previous two simulation results (piecewise and sinusoidal references) the following question arises; why the amplitude of the current input for the piecewise case much larger than the sinusoidal case in spite of that, the amplitude for both desired signal are equal?. This disparity of the current input due to the piecewise case where the piston position has a sudden jump at certain points. Accordingly at these points the slope is unbounded, therefore the HOSMO make a sort of adaptation in order to cover this abrupt change leading to a control effort much larger than that one of sinusoidal case as shown in figures (15) and (21) respectively.

As a matter of fact the control input (the current) is minimal for both type of desired position. It is meant by minimal that the controller, which will be able to regulate the error function $e$ to the origin, will spend just the required effort in order to reject the perturbation term. Canceling the perturbation terms, and then deals with the system as a nominal system and exactly specifying the system response characteristics is the control strategy that leads to minimizing the control effort. Indeed one cannot apply the above mentioned strategy without an exact estimator like the HOSMO.

Moreover the estimation process via HOSMO can be made faster by choosing more appropriate gain matrix $L$ such that $\operatorname{eig}(A-L C)$ are sufficiently far from the right hand side of the imaginary axis.

\section{Conclusion}

A HOSMO based controller design is proposed to improve the piston position tracking for the electro-hydraulic system suffers from parametric uncertainties and unmatched disturbance with only position measured signal. By using only the piston position as a measured output, the HOSMO is designed to estimate both the bounded differentiable unknown form and the states of the phase variable canonical system. Our proposed controller uses the estimated perturbation to cancel its actual effect on system dynamics. Then a simple linear output feedback controller based on pole placement was designed such that the piston position tracks the desired one. Two desired position are considered which are piecewise and sinusoidal where the simulation results have shown an excellent estimations and tracking performance.

\section{References}

[1] Karl Johan Aström and Richard M. Murray, "Feedback Systems", Princeton University Press, 2008.

[2] Leonid Fridman, Arie Levant and Jorge Davila, "Observation of Linear systems with Unknown Inputs via High-Order Sliding-Modes", Taylor \& Francis International Journal of Systems Science, Vol. 38, No. 10, pp. 773-791, 2007.

[3] Yuri Shtessel, Christopher Edwards, Leonid Fridman and Arie Levant, "Sliding Mode Control and Observation", Springer Science+Business Media New York, 2014.

[4] M. L. J. Hautus," Strong Detectability and Observers*", Elsevier Science Publishing Co., Inc., pp. 353-368, 1983.

[5] Miroslav Mihajlov, Vlastimir Nikolić and Dragan Antić, " Position Control of an Electro-Hydraulic Servo System Using Sliding Mode Control", FACTA UNIVERSITATIS, Series: Mechanical Engineering, Vol. 1, No. 9, pp. 1217 - 1230, 2002.

[6] Hong-Ming Chen, Jyh-Chyang Renn and Juhng-Perng Su, " Sliding mode control with varying boundary layers for an electrohydraulic position servo system", SpringerVerlag London Limited, pp. 117-123, 2005.

[7] Cheng Guan and Shuangxia Pan, " Adaptive sliding mode control of electro-hydraulic system with nonlinear unknown parameters", Elsevier Ltd, pp. 1275- 1284, 2008.

[8] Daehee Won and Wonhee Kim, " Disturbance Observer based Backstepping for Position Control of Electro-Hydraulic Systems", Springer International Journal of Control, 2015. 
[9] DaeheeWon, Wonhee Kim, Donghoon Shin, and Chung Choo Chung, " High-Gain Disturbance Observer-Based Backstepping Control With Output Tracking Error Constraint for Electro-Hydraulic Systems", IEEE Transactions on Control Systems Technology, 2014.

[10] Vladimir Miliф, Zeljko Situm and Mario

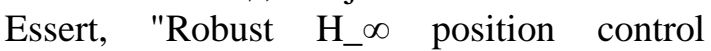
synthesis of an electro-hydraulic servo system", Elsevier ISA, pp. 535-542, 2010.

[11] Francisco J. Bejarano and Leonid Fridman, " High order sliding mode observer for linear systems with unbounded unknown inputs", Taylor \& Francis international Journal of Control, Vol. 83, No. 9, pp. 1920-1929, 2010.

[12] Leonid Fridman, Jorge Davila and Arie Levant, "High-order sliding-mode observation for linear systems with unknown inputs", Elsevier Ltd, 2010.

[13] Sergey K. Korovin and Vasily V. Fomichev, "State Observers for Linear Systems with Uncertainty", Walter de Gruyter GmbH \& Co. KG, 10785 Berlin, Germany, 2009.

[14] J. O'REILLY, "Observers for Linear Systens", Academic Press INC. (London) LTD, 1983.

[15] Jinping Yu, Yuanchun Ding and Falu Weng, " Robust Control for Electro-hydraulic Servo Systems Driven by Doublerod Actuators", IEEE 3rd International Conference on Advanced Computer Control (ICACC), 2011.

\section{Appendix A}

Recall Eq. (3) with $u=0$

$$
\begin{aligned}
& \dot{\gamma}=A \gamma+D \delta\} \\
& y=C \gamma \quad\} \\
& {\left[\begin{array}{c}
y \\
\dot{y} \\
\ddot{y}
\end{array}\right]=\left[\begin{array}{c}
C \\
C A \\
C A^{2}
\end{array}\right] \gamma+\left[\begin{array}{c}
0 \\
C D \\
C A D
\end{array}\right] \delta}
\end{aligned}
$$

From matrices of Eq. (3) we have:

$$
\operatorname{rank}\left(\left[\begin{array}{c}
C \\
C A \\
C A^{2}
\end{array}\right]\right)=3, \quad\left[\begin{array}{c}
0 \\
C D \\
C A D
\end{array}\right]=0
$$

This shows that the relative degree between the output $y$ and the unknown perturbation $\delta$ is three. In other words and from the above procedure, a perturbed linear dynamical system as in Eq. (3) has the strongly observability condition and it has no null dynamic (no invariant zeros). 


\title{
تصميم مسيطر (Output feedback) لمنظومة الكهروـ هايدرولك استنادا الى مراقب النمط

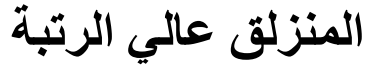

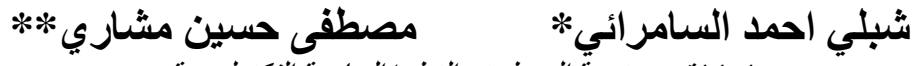

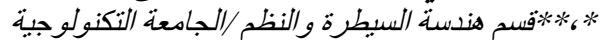

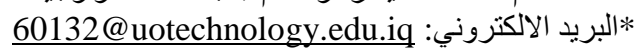

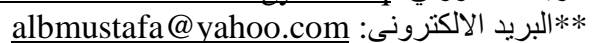

خاصية المر اقبة القوية (Strong observability) للانظمة الخطية المضطربة تؤكد وجود مر اقب ذي نمط منزلق لتخمين مداخل الاضطر اب غير

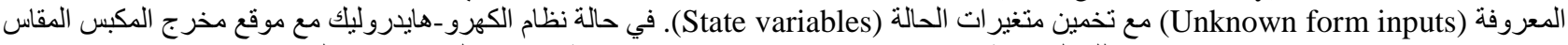
(Strong observability) غير متحققة. في هذا البحث، موقع المكبس و تخمين مشتقاته تستخدم (Piston position measured output)

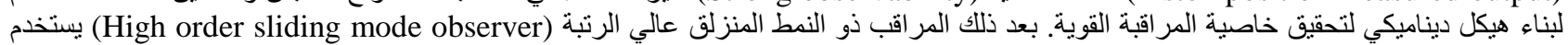

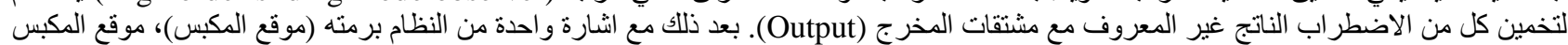

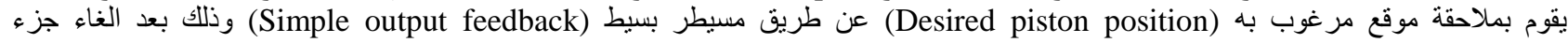

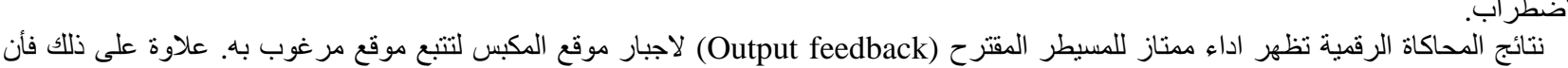

